\title{
G.R.R. Evaluation and Application in IC Manufacturing
}

\author{
Yan-Ju Yu, Yuan Zhou, Tai-Cheng Gong, and Wei-Ting Chien
}

\begin{abstract}
Gauge Repeatability and Reproducibility (G.R.R.) is one part of Measurement System Analysis (MSA), which is to appraise the variability of measurement system. There are three methods to evaluate G.R.R.: Range, Average-Range and ANOVA. Range method is only to approximately evaluate measurement variability but cannot estimate the contribution of repeatability and reproducibility. So, it's not applicable in IC manufacturing. In this paper, we studied and analyzed the difference between Average-Range and ANOVA in G.R.R. evaluation by using IC manufacturing data.
\end{abstract}

Index Terms-G.R.R., average-range, ANOVA, NDC, IC manufacturing.

\section{INTRODUCTION}

MSA is very important to appraise the quality of a measurement system, especially for IC manufacturing with an advanced technology node, i.e. 40nm and below. The measurement error may be from appraiser, equipment, material, method or environment. The variation source analysis for the measurement system is more and more inevitable.

In MSA, G.R.R. [1], [2] as the appraisement of measurement system precision can be evaluated by the following three methods [3]: Range, Average-Range and ANOVA (Analysis of variance). Range method is only to provide a quick approximation of measurement variability and cannot calculate the repeatability and reproducibility, so Average-Range and ANOVA are more popular in IC manufacturing. Average-Range method [4], [5] is using the average and range value to appraise the measurement system, while ANOVA method [6], [7] is using the standard deviation. In this paper, we studied and analyzed the difference of Average-Range and ANOVA and the application in IC manufacturing.

\section{MethodOLOGY}

In this study, no manual operation was adopted and all data were collected by automatic metrology tools. And we compared G.R.R. data derived from Average-Range and ANOVA method for thick and thin films.

\section{A. Methodology for G.R.R. Evaluation}

Average-Range and ANOVA have different statistical calculation, as shown in Table I. The former uses the average range by a constant $(\mathrm{K})$ to calculate the $\mathrm{EV} \& \mathrm{AV}$, while the latter uses the standard deviation. In addition, to estimate

Manuscript received April 29, 2015; revised August 5, 2015.

All authors are with the Corp. Quality and Reliability Engineering unit, Semiconductor Manufacturing International Corporation, Shanghai, China (e-mail: Salus_Zhou@smics.com, Kevin_Gong@smics.com, Lisa_Yu@smics.com, Kary_Chien@smics.com).
G.R.R., the process variation should be determined. According to MSA manual, there are 3 approaches [3] to have the process variation. The first one is to use specification tolerance. The resultant G.R.R. is also called Precision/ Tolerance $(\mathrm{P} / \mathrm{T})$ ratio, which is the most popular method used in IC manufacturing. The second one is to use surrogate process variation in the case of no sufficient samples to represent the process variation. That is, a long-term inline process variation taken from actual production performance is adopted. Here we use the actual standard deviation of inline or offline SPC charts which is recorded as inline TV (total variation). The last one is to use process variation taken from the parts in the G.R.R. evaluation itself by summing the square of both the repeatability variation, reproducibility variation and the part variation.

Here we also introduce NDC (Number of Distinct Categories) as another index to appraise metrology tool's capability that how many data groups could be clarified. It is the number of non-overlapping 97\% confidence intervals that will span the expected product variation and should be greater than or equal to 5 [3].

TABLE I: G.R.R. DiFFERENCE CALCULATION METHODS FOR AUTOMATIC MEASUREMENT SYSTEM

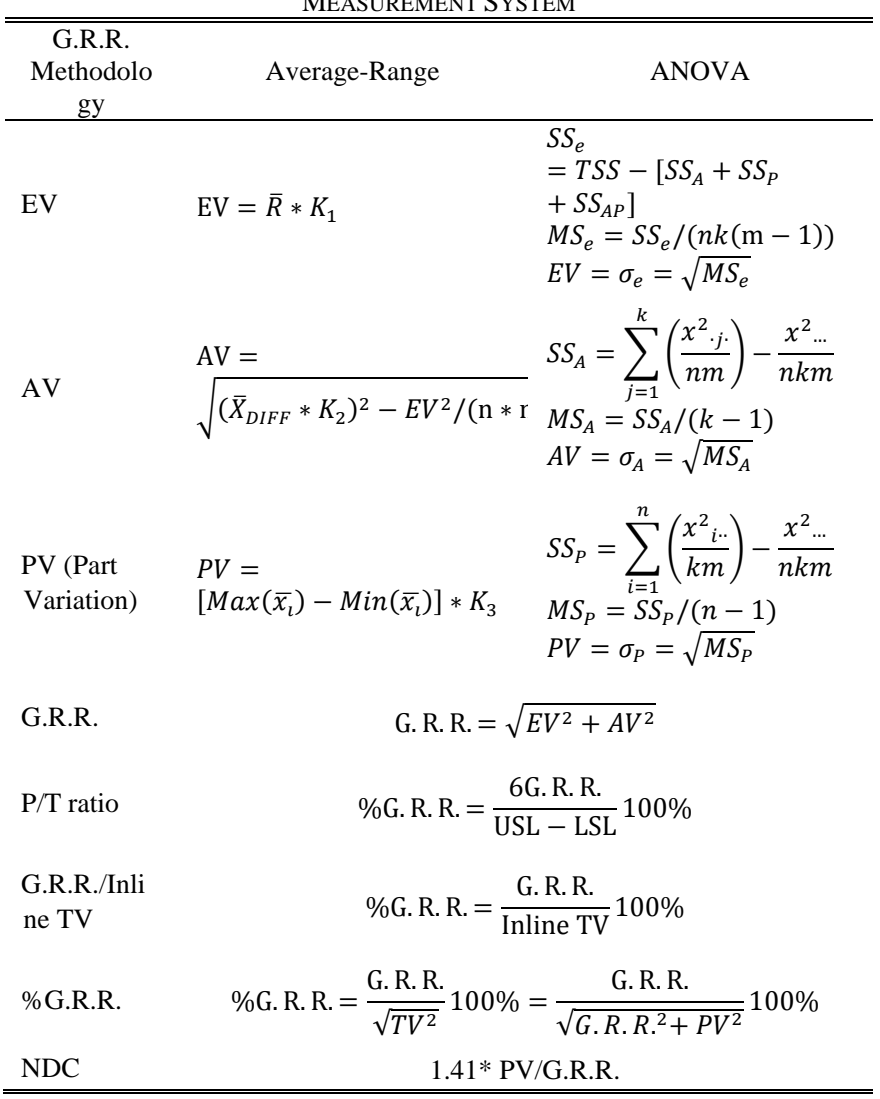

B. Experiment Design for G.R.R. Evaluation

We designed 3 groups of experiments as listed in Table II 
to study the effect of measurement objectives, measurement and estimation methods on G.R.R. Additionally, Precision/ Tolerance $(\mathrm{P} / \mathrm{T})$ ratio is also estimated by using the data.

TABLE II: EXPERIMENTS FOR G.R.R. EVALUATION

\begin{tabular}{llll} 
& Wafer & Operator & Times \\
\hline Average-Range & $1 \mathrm{pcs}$ & 3 & 3 \\
Average-Range & $5 \mathrm{pcs}$ & 3 & 3 \\
ANOVA & $5 \mathrm{pcs}$ & 3 & 3 \\
\hline \hline
\end{tabular}

\section{DATA ANALYSIS AND RESUlTS}

Based on above design, typical thickness metrology tools are selected for data collection, covering the thick and thin film thickness of production line.

\section{A. Different G.R.R. Methods Data Analysis}

1) $\mathrm{P} / \mathrm{T}$ ratio

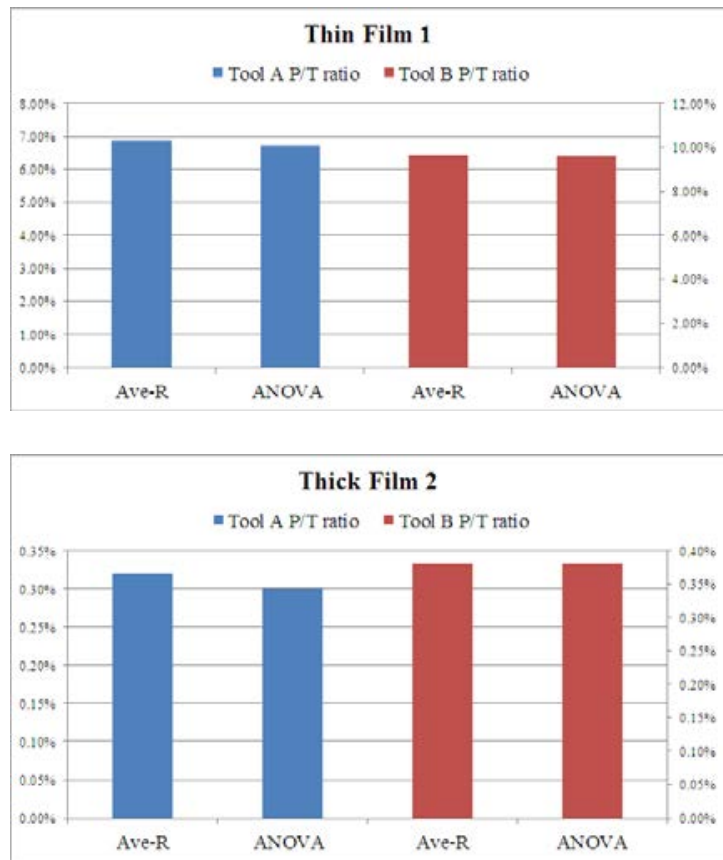

Fig. 1. $\mathrm{P} / \mathrm{T}$ ratio comparison.

From Fig. 1, it can be seen, whatever thick or thin film, P/T ratio derived from Average-Range is higher than that derived from ANOVA.

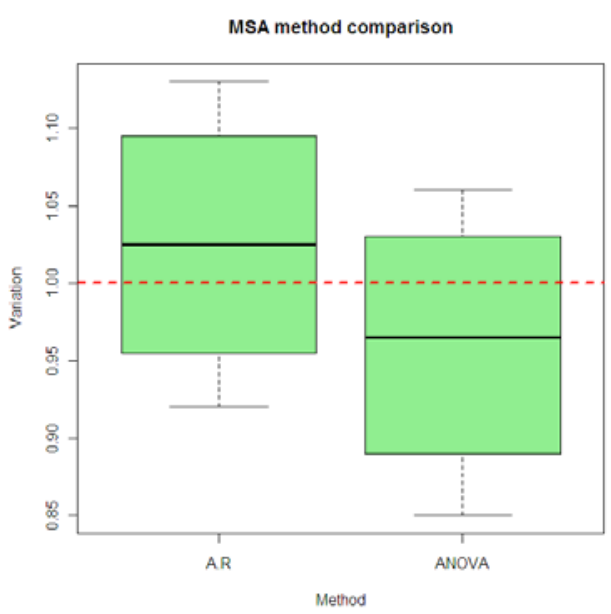

Fig. 2. Two methods calculation result comparison.

To validate the observation, we calculate the standard variation derived from Average-Range and ANOVA, respectively, by repeated sampling from a group of normal distributed samples with standard variation of 1 . It can be seen from Fig. 2, the mean value of Average-Range is higher than that of ANOVA, although t test shows no statistical significant difference with $\mathrm{p}$ value of 0.34 which is higher than 0.05. In this case, Average-Range is preferred considering its friendly calculation.

\section{2) PV}

Measurement system error has not only EV and AV, but also part variation.

In this experiment, limited by sample preparation, only within wafer and wafer to wafer variation are counted. As shown in Fig. 3, PV extracted from this experiment (both Average-Range and ANOVA) cannot represent inline production measurement for both thin and thick films. It's indicated lot-to-lot or tool-to-tool variation cannot be neglected for this process. Therefore, to have a relative accurate G.R.R. estimation, sample preparation should cover all possible variation sources to total part variation. However, it's not applicable in real operation in G.R.R evaluation.

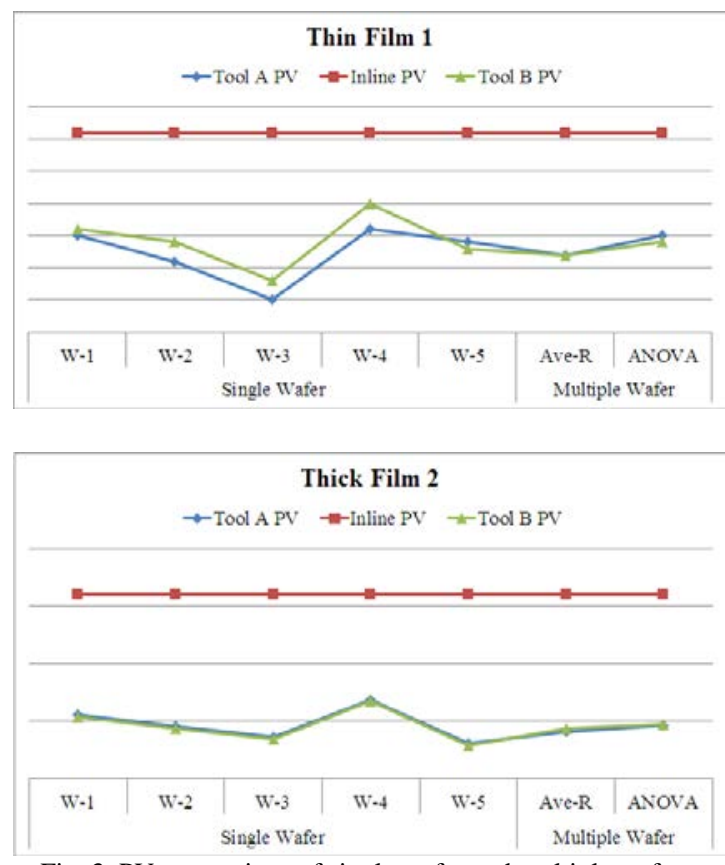

Fig. 3. PV comparison of single wafer and multiple wafers.

3) Effect of process variation on G.R.R.

Using the three approaches described in section 1, \%G.R.R. is calculated as shown in Fig. 4. It can be seen G.R.R./TV is much higher than the other two approaches because of the under-estimated TV (only within wafer and wafer-to-wafer variation is covered limited by sample preparation). And P/T ratio is lower than G.R.R./inline TV. Additionally, from the comparison of inline TV derived G.R.R./inline TV and P/T ratio. The process capability can be estimated. The higher the ratio of inline G.R.R./inline TV to $\mathrm{P} / \mathrm{T}$ ratio is, the better the process capability is. Obviously, the process capability of thick film is much better than that of thin film.

In addition, it's noted that thin film measurement is challenging for both tool $\mathrm{A}$ and $\mathrm{B}$. Both $\mathrm{P} / \mathrm{T}$ ratio and G.R.R./inline TV is marginal pass comparing with $10 \%$ criteria. Checked the stability monitor and production line monitor data of the same level for thin film, it's found the contribution of metrology tool induced variation is about $15 \%$ of total process variation 
$(\sigma($ metrology $) / \sigma($ process $)=0.022 / 0.1435=15 \%)$, and higher than $10 \%$ (Fig. 5 and Fig. 6). Further checked the metrology tool SPEC sheet limit, it's 0.017. It means the thickness measurement tool is not suitable for the thin film measurement due to the variation of the thin film measurement is out of the metrology tool limitation $(0.022>$ 0.017).

From the above discussion, both $\mathrm{P} / \mathrm{T}$ ratio and G.R.R./inline TV can effectively evaluate metrology tool capability. To simplify G.R.R. evaluation, $\mathrm{P} / \mathrm{T}$ ratio is preferred. That should be the reason for $\mathrm{P} / \mathrm{T}$ ratio is so popular.

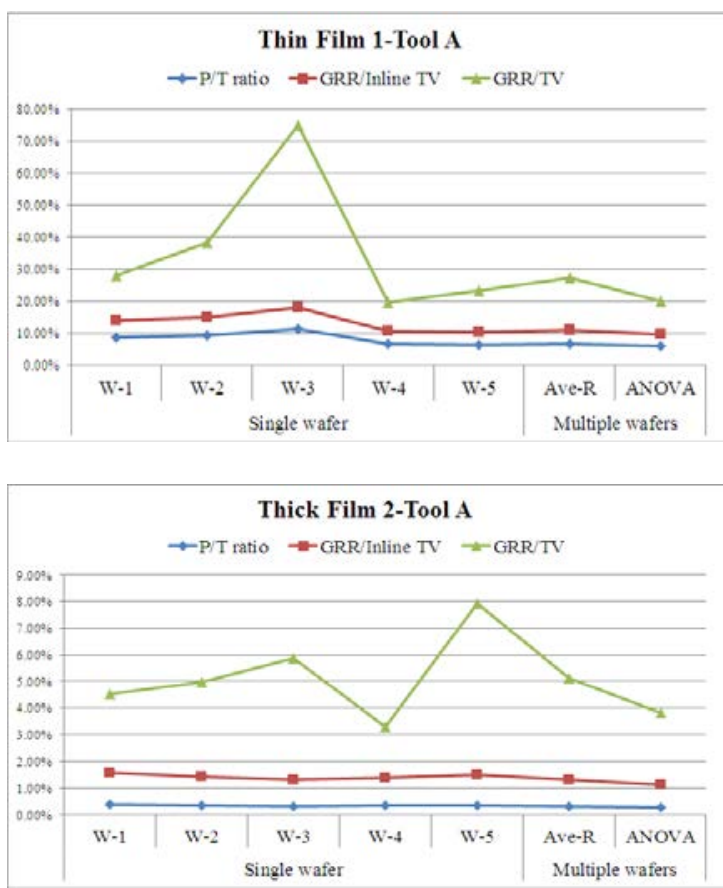

Fig. 4. P/T ratio, GRR/inline PV and GRR/TV comparison.

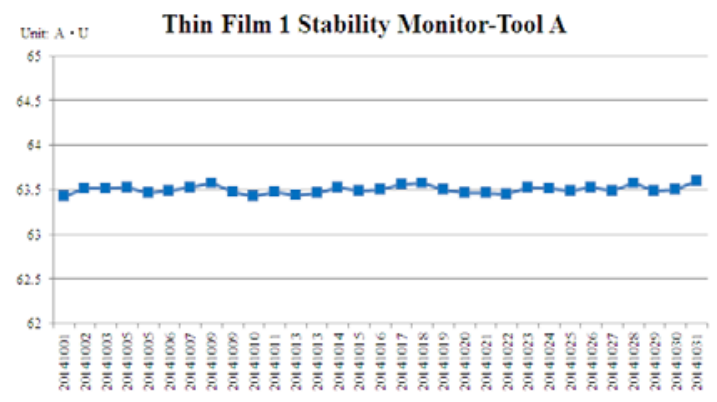

Fig. 5. Tool a thin film 1 stability monitor performance.

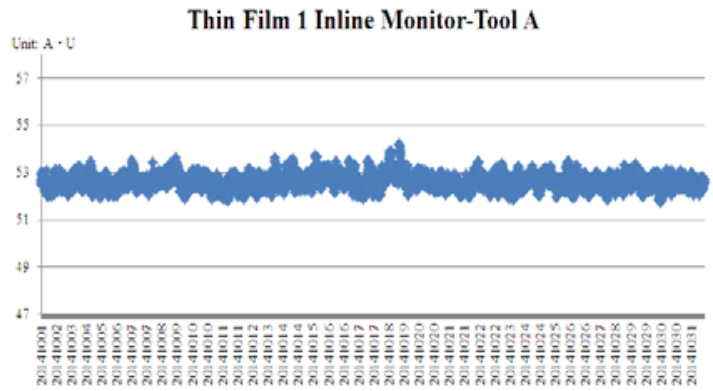

Fig. 6. Tool a thin film 1 production line monitor performance.

4) Number of Distinct Categories (NDC)

To further evaluate the capability of measurement system relative to the parts variation, NDC is calculated, as shown in
Fig. 7. It can be seen, the thinner the film is, the lower NDC is, and even lower than 5. It's reasonable, as discussed previously. Thin film has a relative high G.R.R. but low PV, comparing with thick film, as shown in Fig. 1 and Fig. 2. Especially in the case of NDC lower than 5, it's challenging to measure thin film using the same metrology tools as thick film.

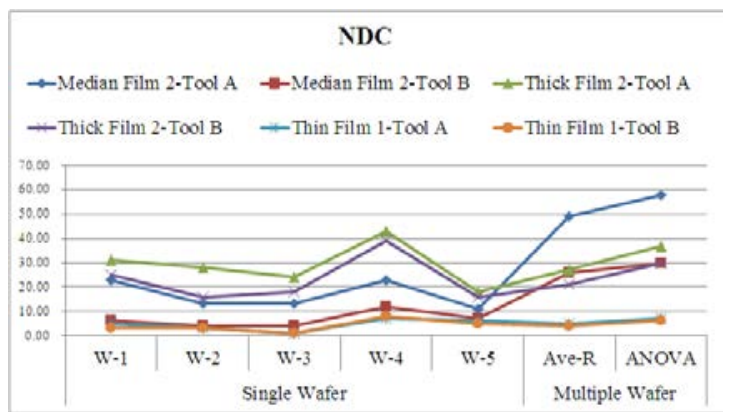

Fig. 7. NDC comparison.

\section{CONCLUSION}

In this paper, we studied the effect of statistical analysis methods (average-range and ANOVA) on G.R.R. estimation by using the data measured by the typical thickness metrology tools. No statistical significant difference is found in G.R.R. by Average-Range and ANOVA methods.

As for \%G.R.R characterization, we applied 3 approaches to determine process variation, that is, surrogate process variation by long-term inline or offline production monitor, specification tolerance and the variation from G.R.R test. Owing to the complex IC manufacturing process, the sample preparation in G.R.R. test is limited and the derived variation could hardly represent that of production line and is under-estimated comparing with long-term production line variation. So this approach is not preferred. However, from the demonstration result of thin, median and thick film data, SPEC tolerance derived from $\mathrm{P} / \mathrm{T}$ ratio has the same sensibility to metrology tool capability as surrogate process variation derived from G.R.R./inline $\mathrm{TV}$, and $\mathrm{P} / \mathrm{T}$ ratio is directly linking to customer requirement besides its operation friendly (SPEC tolerance kept unchanged, but inline monitor derived from surrogate process variation is dynamic). Therefore, $\mathrm{P} / \mathrm{T}$ ratio is preferred and popular in IC manufacturing.

Additionally, from the comparison of inline variation derived from G.R.R./inline TV and P/T ratio, the process capability can also be estimated. The higher the ratio of G.R.R./inline $\mathrm{TV}$ to $\mathrm{P} / \mathrm{T}$ ratio is, the better the process capability is.

As for the metrology tool with $\mathrm{P} / \mathrm{T}$ ratio closing to $10 \%$ criteria, the measurement is very challenging for the measurement target. The risk can be further accessed using the stability monitor data of the target measured by the metrology system. So the routine measurement tool stability monitor is also important.

\section{REFERENCES}

[1] L. B. Barraentine, Concepts for R\&R Studies, Milwaukee, Wisconsin: ASQ Quality Press, 1991, ch. 1, pp. 2-4.

[2] J. Mandel, "Repeatability and reproducibility," Journal of Quality Technology, vol. 4, no. 2, pp. 74-85, 1972. 
[3] AIAG Editing Group, Measurement System Analysis (MSA) Reference Manual, 4th ed. 2010, ch. 3, pp. 92-120.

[4] D. C. Montgomery and G. C. Runger, "Gauge capability analysis and designed experiments. Part I: Basic methods,” Quality Engineering, vol. 6, no. 1, pp. 115-135, 1993.

[5] S. B. Vardeman and E. S. VanValkenburg, "Two-way random-effect analyses and gauge R\&R studies,” Technometrics, vol. 41, no. 3, pp. 202-211, 1999.

[6] D. J. Wheeler and R. W. Lyday, Evaluating the Measurement Process, 2nd ed. Knoxville, Tennessee: SPC Press, 1990, ch. 2, pp. 22-28.

[7] P. Tsai, "Variable gauge repeatability and reproducibility study using the analysis of variance method," Quality Engineering, vol. 1, no. 1, pp. 107-115, 1998.

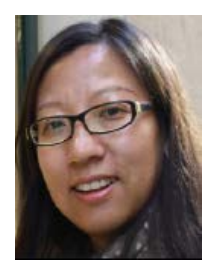

Yan-Ju Yu graduated as a Ph.D. candidate from the School of Material Science in Harbin Institute Technology in 2003. She has worked in semiconductor manufacturing industry for more than 10 years, focusing on device level reliability, metrology tools MSA, raw materials quality control, supplier management etc. She is one of senior managers in quality and reliability engineering unit of Semiconductor Manufacturing International Corporation (SH).

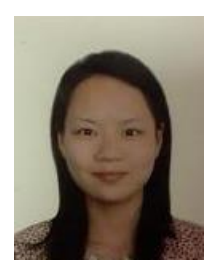

Yuan Zhou was born in Shanghai, China, in October 1985. She obtained the master degree in industrial engineering in 2010. Now she focuses on the research of metrology calibration and MSA as a senior calibration engineer in Semiconductor Manufacturing International Corporation.

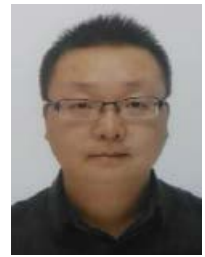

Tai-Cheng Gong was born in Hunan, China, in August, 1982. He obtained the bachelor degree in industrial engineering in 2005. Now he is calibration assistant manager in Semiconductor Manufacturing International Corporation, and focuses on the research of production line measurement system analysis and improvement in wafer foundry.

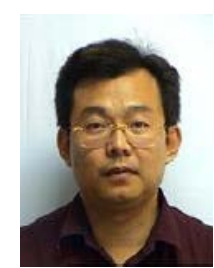

Wei-Ting Chien was born in Taiwan in 1965. He received his Ph.D. degree in industrial engineering from Texas A\&M University in 1994. Before he came back to Taiwan for industrial jobs in semiconductor manufacturing in 1995, he did post-doc researches with HP, and IBM for a year on software reliability modeling, and highly reliable system optimization, respectively. After being with Nan-Ya Technology Corporation, Intel, and TSMC, he joined SMIC in Shanghai in 2001. In 2010, he was deputy operation director with STMicroelectronics in Singapore. He re-joined SMIC in 2011 as Corp Q\&R Vice President until now. His current assignments cover business process reengineering; quality system management; in coming, in-process, and outgoing quality control; subcontractor quality; reliability engineering; process and product qualification; failure analysis; and ESH (environment, safety, and hygiene). He has published over 170 papers, more than 60 patents, and is senior member of IEEE, member of Phi-Tau-Phi Scholastic Honor Society, and a member of Shanghai 1000-Person Plan. 